\title{
RESEARCH Non-verbal Skills: Unavoidable in Communication
}

\author{
Yog Raj Lamichhane
}

\section{ABSTRACT}

KEY WORDS

Communication

Verbal Communication

Non-verbal Communication

Facial Expression
This paper aims to define communication and incorporate differences between verbal and non-verbal communication. The special focus is given to different kinds of non-verbal skills with their color and creed. Their contribution in professional field is critically examined. As communication is an ongoing and complex process of transmission and reception of message, the implication of non-verbal communication is indispensable. When verbal and non-verbal cues are in conflict, verbal skills are virtually disregarded and the meaning and implication of 'what' heavily depends on non-verbal signs and symbols. The paper also highlights the different forms of vocalic and non-vocalic nonverbal cues with rational categorization. It also explores space in which culture and gender affect non-verbal communication by creating different meanings of particular item of behaviors. In this way, this paper attempts to excavate the importance of non-verbal communication in professional life.

A single conversation with a wise man is better than ten years of study.

\section{Chinese Proverb}

WHEN WE GO TO THE history of the word communication, it is defined as derived from a Latin word Communis which means "to share", the word 'Communication' means sharing of ideas, concepts, messages and words expressed through a language which is easily comprehensible to the listener (Kaul, 2009 p.3). In this definition; communication is presented as transmission and reception of a message in comprehensive way where language plays significant role in the process. From the very beginning of the communication as interdisciplinary subject, verbal and non-verbal skills have been simultaneously used. In the early 1940s and 1950s communication as an interdisciplinary subject began to gain ground. In the initial phase, public speaking was emphasized and extensive study on non-verbal as well as the verbal cusses was conducted (Kaul, 2009, p.4). Communication includes both verbal and non-verbal sign and symbols deliberately or unconsciously used in transmission and reception of meaning. Simply, communication is known as sharing of information, ideas, emotion, knowledge, etc. In different professional career including banking, information science, publication and accounting, one needs communication skills as prime quality. In extended 
sense, it is not only the exchange of ideas and information but it is also the transfer of meaning.

The idea that there are clear boundaries between verbal and non-verbal communication and that it is possible to distinguish sharply between linguistics and nonlinguistic features of conversational events is rooted more in our own logical and methodological assumptions than in the psychological realities of face to face communication (Arndt and Janney 1987, p.92). Both verbal and non-verbal communication make the cycle of communication complete. It is more applicable in face to face communication. In this sense, there is no verbal or non-verbal, but only communication. It is not in favor of separating verbal communication and non-verbal communication. The ides supports the proper combination of both forms of communication. It counters the myth that communication can only be done through the words which is verbally presented in oral or written form. It generates an extended space for non-verbal communication to counter the argument of classical concept of communication. Communication is behavior and by using it any group mediates and sustains its relationship. The relationship and state for the participant can be simply identified by the flow of communication. People sustain in their societies through communication. In this sense, it is an art of interaction.

Good communication skills are valued as basic requirement in job market. While selecting anyone as employ in job market, the communication plays very influential role (Curtis, 1989, p.45). He also associates that $42 \%$ who responded concurred that most valued in the contemporary job market and communication skills.

\section{Why Non -Verbal Communication?}

A strong confident person can rule the room with knowledge, personal style, attitude and great posture.

\section{Cindy Ann Peterson}

Non-verbal communication is a process of generating meaning using behavior other than words. Non-verbal communication includes all communication other than language (Andersen, 1999, p.2). The statement signals the nonlinguistic segment of communication. Beyond the linguistic or grammatical rules, for the communication competence, therules ofnon-verbalcommunication of target language should be understood firstly for good communication. It includes all messages which are communicated other than words that both parties of communication exchange. It creates comfort in communication by avoiding ambiguity and generating common ground for understanding. Non-verbal communication also helps to avoid ambiguity and provide feedbacks. For example, nodding one's head and saying 'ub-hub' signals understanding on behalf of the listener. The use of non-verbal communication can also facilitate "common ground" by allowing speakers and listeners to monitor and signal the extent to understanding of a communication context is being shared (Clark and Brenna, 1991, p.127).

Naturally, in which way we walk, we talk, we eat and we posit ourselves give meaning then what we actually say in different situations. With the close observation of some one's walking, talking and positioning of hands, we can get hold of utmost meaning there. Non-verbal statement includes the tone, loudness, speed, and timing of the words used in particular situation, but it does not include words and their associated connotation. Thus, when communication takes place, in a face-toface context, it can convey than just words. But the same non-verbal communication is varying depending on the age, sex and various cultural 
involves (Harris, 2002, p.155). In this sense, same non-verbal communication has plural meaning in multiple cultures. Some researchers have argued that non-verbal communication is deliberate and strategic; there is recent evidence that even strategic behaviors can occur automatically (Larkin \& Chartrand, 2003, p.134). As Larkin says, we use non-verbal skills of communication strategic and in deliberate way but he even highlights the deliberateness of strategic performance of communication which happens automatically. It advocates the non-intentionality of non-verbal communication. Simple silence is also considered as speech. It is understood that silence delivers non-verbal cues related to different communication situation where we are. In this way, non-verbal communication is indispensable in communicative process. Even the problem which we have to face in cross cultural communication can simply be solved by means of non-verbal tools.

\section{Verbal versus Non-verbal Communication}

Nothing in life is more important than the ability to communicate effectively.

\section{Gerald R. Ford}

In language system, verbal and non-verbal communication work side by side. Especially while working with second language as well as in intercultural interaction, the dependency to nonverbal communication increases strangely. The fact that at least one communicator is working in a second language means the verbal content may not be clear as it would be in an intercultural interaction. Consequently, the reliance on nonverbal communication may be ever greater than normal (Singelis1994, p.275).In association to non-verbal cues verbal cues are disregarded to disclose the maximum reality It can be seen that the non-verbal had more effect than the verbal contents, in fact about five times as much; when the verbal and non-verbal messages were in conflict, the verbal contents were virtually disregarded (Argyle, 1972).

Non-verbal cues used by any speaker are more powerful to impress others than only verbally transferred materials. It is extra effective in encountering a personal at first time. In the absence of close coordination between verbal and non-verbal communication, the non-verbal skills stand there as dominant factor to be considered. For example, first impression involves facial expression; even without the verbal description non-verbal cues like facial expression denote the real meaning through behaviors performed by face in certain situation (Willis \& Todorov, 2006, p.592). Excluding verbal signs and symbols any behavior performed by face can be included in facial expression. It advocates the role of nonverbal skills as means to reflect the internal psyche of any character which have involvement in social interactions. In this manner focusing only on words leads communication to malfunction.

The pedagogical significance of the non-verbal communication cannot be undermined. Actually the success of both teachers and students depend upon the effective communication between in the class, but becomes handicapped without the proper use of non-verbal communication (Woodfolk, 1983, p.103). The writer with the pedagogical implication of non-verbal communication tries to designate the real strength of it. Non-verbal communication, which is more deliberate and highly applied in oral communication than literacy. Especially in face to face situation, dramatically maximum part of communication is done by means of non-verbal symbols. According to one authority $7 \%$ of meaning in a face to face situation is derived from word symbols, 38\% from voice tone and inflections and $55 \%$ of the meaning in 
face to face communication is transferred through non-verbal symbols (Lesikar \& Pettit, 2004, p. 646).Here maximum meaning in face to face context is covered by such non-verbal signs and symbols.

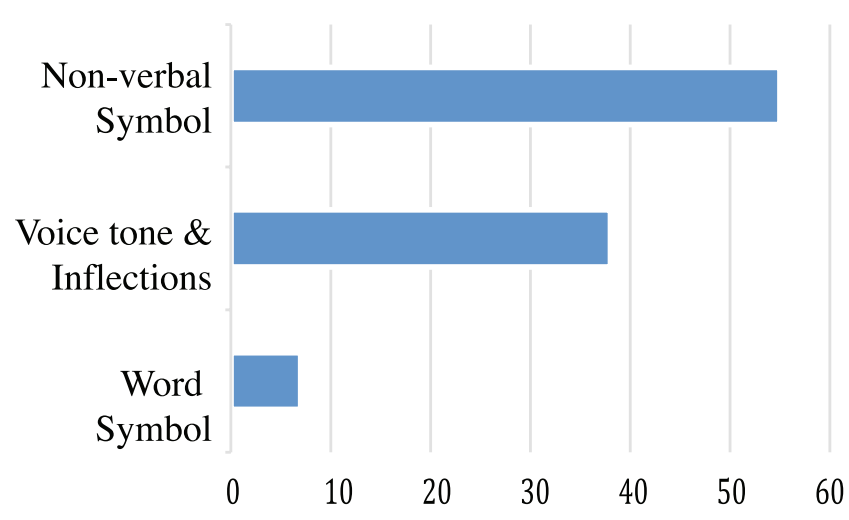

Figure 1: Communication in Face to Face Situation

\section{Color and Creed of Non-verbal Communication}

The most important thing in communication is hearing what isn't said.

\section{Peter F. Drucker}

Face as one of the most important site for communication is highly noticeable in face to face interaction. It is the primary source of information next to human speech for this reason and because of the faces visibility we pay a great deal of attention to the message we receive from the face of other (Knapp and Hall, 2006 p.260). This places the role of face to create total meaning. Utmost concern is given to face and we consider the face as foremost foundation of human non-verbal communication. There is multiplicity of facial expression because of its maximum visibility.

In some cultures, there are socially prescribed rules about covering the face, but in the Western culture the best we can do to make it less visible is to wear spectacles of some glasses (Birdwhistell, 1970). Such plural possibilities of facial expression create challenges to audiences to construct meaning. The sense of so many gestures and movement are complicated, a few of the meanings are explicit, many of them are only self-expression, and some reflect personality and attitude. So the classification of body behavior is complex. Audiences have to face problem especially decoding such expressions. Regular practice leads to better understanding. Possibly one of the most comprehensive and scientific categorization of non-verbal communication is offered by Zoric and Pandzic.

1. Chronemics - Timing of verbalizations and pauses

2. Haptics - Contact and deliberate touch between individual.

3. Kinesics-All forms of body language and body movement, including facial expressions, eye movement, gesture, and posture

4. Oculesics - Intentional and unintentional eye contact in the act of communication

5. Olfactics - The influence of odor

6. Physical Appearance - Characteristics of the body, clothing, hairstyle, etc

7. Proxemics - Consideration of personal space and arrangement of physical items

8. Silence - The absence of verbal and nonverbal communication

9. Symbolism - Meaning associated with symbols

10. Vocalics-Vocal impacts on the act of speaking, to include tone of voice, timbre, volume, and rate of speech (Zoric \& Pandzic, 2007)

The authors have done a useful and broad categorization of non-verbal communication which is somehow impossible to reject. The 
categorization is so effective because of simple description by the side of them. The indispensable ingredients of non-verbal communication are understood such things as body movements, space, time and voice modulation as well as universal characteristics of the environments, color and layout.

Various researchers on non-verbal communication have distinguished different kinds of nonverbal skills relating to our potentiality. They are classified as vocalic and non-vocalic. The vocalic part of non-verbal communication also play significant role in communication. Nonverbal communication like, paralanguage consists of variation in speech, such as voice quality, volume, tempo, pitch, non-fluencies (for example uh, um, ah) laughing. Humming or filling silence with sound like ummm or aaaa and intonation and modulation that accompany speech add for the better communication as paralinguistic pact. Especially while one has to know the special subjective feeling such non-verbal cues are so useful. In communication, only limited percent of meaning is supplied by actual words themselves because they may have different interpretations. To keep away from such multiple interpretations, it is so vital to pay attention not only to what is being said, but how something is being said. There, wherever the correct implication of the communication can be found. At this point manner of telling something is so vital than what is being told. In this way voice inflections like tone, speed, emotions, space, volume, etc. are concerned. While words being spoken, the meaning can be dramatically changed because of the style of pronunciation. Eye gaze, eye contract, deictic, gesticulation, and proxemics are so common non-vocalic cues in frequent uses. Ekman and his associates have identified five typical types of gestures which are familiar in communication:
1. Emblems - Ok gesture; the meanings are known by most members of social group and are used intentionally to convey certain message

2. Illustrative - When we want to show that the object is round and describe a circle by hand

3. Facial Expression - Which express our emotional state

4. Adjusting gestures - They help us in maintaining and controlling interaction with interlocutors.

5. Adapting gestures - Those stereotypical gestures, we make in condition of mental tension (Ekman, 2002, p.23)

The gestures contribute to the understanding process along with the support of artifact, dress, and fashion worn by a person. These contribute while getting meaning in social setting much. And, the culture differences in non-verbal communication can also be realized in gesture. It conveys more emotional and touching connotation than verbal communication

Such as being late or early, keeping others waiting and other relationships between time in chronemics, personal space of body distance as proxemics, interpretations of non-verbal clues, related to hand and arms gestures in gesticulation, locating something in deictic and eye contract and different types of gaze are major non-verbal cues which are non- vocalic. For example: shifting eyes contract is understood as lack of concentration and open hands and arms, especially extended, could indicate a receptive participant. Our arrangement of four personal spaces delivers different meaning as suggested by Anthropologist -Hall.

1. Intimate - Physical contact to 18 inches

2. Personal - 18 inches to 4 feet 
3. Social - 4 feet to 12 feet

4. Public -12 feet to range of eye sight and hearing (Hall, 1966, p.107)

Thus, the sitting arrangement in any meeting is very important. The space is major source to supply sense here. Different space patterns provide different meaning in speakers' and listeners' mind. For the effective communication both listener and speaker requires good understanding of verbal and non-verbal behavior which are contextual as well as cultural. They have neither strongly structured foundation nor rigid formula but supported by normative rules. They are so hurried to surpass cultural boundaries. These four different levels of space are realized in many cultures but differently. For example, Latin American tends to interact more closely than the students of European background (Foreston \& Larson, 1968, p.109). Arabs, at least in the past, learned to interact with others at distances close enough to feel others person's breath (Hall, 1963, p.1003). In comparison to German, Italians interact more closely than either Germans or Americans. Colombians were found to interact at closer distances than Costa Rican (Shutter, 1967, p.46). The proxemics deal with the study of space elements, such as distance between people and objects and its meanings are diverse in different backgrounds.

\section{Conclusion}

Communication is an ongoing process of transmission and reception of message. It is more than the transfer of ideas and information and it aims to convey the meanings. The real communication

\section{REFERENCES}

Andersen,P.A.(1999).Non-verbalcommunication: Forms and Functions. Mountain View, CA: Mayfield Publishing Co. is done through verbal and non-verbal signs and symbols, deliberately or unconsciously. When verbal and non-verbal communications are in conflict, verbal skills are virtually disregarded. Because of that, communication is not only science but it is an art, and it should be developed by all professional personalities and common communicators for maximum transfer of meanings in communication process. In every communication, focusing only to words is major problem. Non-verbal communication consists of vocalic elements and non- vocalic elements. In the absence of knowledge related to non-verbal communication, no communicator transfers message and meaning clearly and no receiver detects such message and meaning significantly. Non-verbal communication does not perform perfectly in isolation. For better communication, both verbal and different types of non-verbal skills work simultaneously in communication process. In the absence of appropriate knowledge of non-verbal skills, it may lead to malfunction of communication. Some non-verbal skills have universal meanings where as some cues carry different meanings in different cultures. The problem which we have to face in cross cultural situation can be minimized by means of nonverbal communication. Especially in face to face situation, dramatically maximum meaning is transferred by means of non-verbal skills and they are unavoidable. And all these skills are being agent of powerful presentation in professional life. Ultimately, they make a better environment in meaning making process.

Argyle, M.(1972). ThepsychologyofInterpersonal Behavior, Harmonds Worth: Penguin. 
Arndt, H., \& Janney, R. W. (1987). Inter Grammar: Toward an integrative model of verbal, prosodic and kinesic choices in speech. Berlin: Mouton de Gruyter.

Birdwhistell,R.L.(1970). Kinesics and context. Essay in Body motion communication. Harmondsworth:Penguin.

Clark, H., \& Brennan, S. (1991). Grounding in Communication. In L. Resnick, J. Levine and S.Teasley (Eds.), Perspectives on Socially Shared Cognition (E. Churchill, D. Snowdon, and J. Munro, eds). Washington, DC: American Psychological Association, p. 127-149.

Curtis, D. B., Wensor, J. L. \& Stephans, R. D. (1989). National preferences in business and communication education. Communication Education, 45, p. 40-57.

.Ekman, P., Wallace, F., and Joseph, H. (2002). Facial Action Coding System, Salt Lake City, USA, p. 23.

Foreston, R. F., \& Larson, C.U. (1968). The dynamics of space: An experimental study in proxemic behavior among Latin Americans and North Americans. Journal of Communication. 18, p. 109-116.

Hall, E. T. (1963). A system for the notation of proxemic behaviors. American Anthropologist, p. 1003-1026.

---. (1966). The Hidden Dimension. Garden City N.Y.: Daubleday, p. 107-122

Harris, T. E. (2002). Applied organizational communication: Principles and programmatic for future practices. London; Lawrence Erlbaum Association published. p. 155.
Kaul, A. (2009). Business Communication: CPHI Learning Private limited Delhi, Second Edition, p. 3.

Knapp, M., \& Hall, J. (2006). Non-verbal communication in human interaction. Belmont, CA: Thomson Wadsworth.

Lesikar, R. V., Pettit, J. D. (2004).Business communication: Theory and Application. A.I.T.B.S Publisher and Distributer, Krishna Nagar, Delhi. Sixth Edition, p. 646.

Shutter, R. (1967). Proxemics and Tactility in Latin America. Journal of communication, p. 46-52.

Singelis, T. (1994). Non-verbal communication in intercultural interactions. In R. Brislin \& T. Yoshida (Eds.) Improving intercultural interactions, Thousand Oaks, CA: Sage.p. 268-294.

Willis, J., \& Todorov, A. (2006). First Impressions: Making Up Your Mind

Exposure To A Face. Psychological Science, 17(7), 592-598.

Woodfolk, A\& Brooks, D. (1983). The influence of teachers' non-verbal behavior on students' perceptions and performance. In Gorden E. (Ed), Review of Research in Education Washington D.C. Vol 10, p. 103-141.

Zoric, G., Smid, K., \& Pandzic, I. S. (2007). Facial Gestures: Taxonomy and applications of non-verbal, non-emotional facial displays for embodied conversation agents. In $\mathrm{T}$. Nishida (Ed.), Conversational Informatics: An Engineering Approach. West Sussex: John Wiley \& Sons Ltd. 
Yog Raj Lamichhane is an Assistant Professor in English at School of Business, Pokhara University, Nepal. He has five years of experience in teaching.
His area of interests in research includes teaching communication

e-mail:lcyograj@gmail.com 